\title{
High Energies and Radiation Effects
}

\begin{abstract}
In this chapter we characterize the high-energy spectra of protons that can penetrate shielding and determine the radiation dose to humans and equipment in space. High-energy spectral breaks or "knees", seen in all large SEP events, determine the contribution of highly penetrating protons. The streaming limit, discussed earlier, places an upper bound on particle fluences early in events and the radial variation of intensities is important for near-solar and deep-space missions. The streaming limit is a strong function of radial distance from the Sun. We also consider requirements for a radiation storm shelter for deep space, a mission to Mars, suitability of exoplanets for life, and radiation-induced chemistry of the upper atmosphere of Earth.
\end{abstract}

We must recognize that solar energetic particles (SEPs) are of more than scientific interest. They can be a serious radiation hazard to astronauts and equipment in space beyond the protection of Earth's atmosphere and magnetic field. Protons of $\sim 150 \mathrm{MeV}$ can penetrate $20 \mathrm{gm} \mathrm{cm}^{-2}(7.4 \mathrm{~cm}$ ) of $\mathrm{Al}$ or $15.5 \mathrm{~cm}$ of water (or human flesh). Such protons are considered to be "hard" radiation, in that they are very difficult to shield, and they are orders of magnitude more intense than the GeV protons that define a ground-level event (GLE). Most of the other radiation risk to humans in space from SEP events comes from protons in the energy region above about $50 \mathrm{MeV}$, or "soft" radiation. This is where protons begin to penetrate spacesuits and the skin of spacecraft. Further studies of radiation dosage and engineering design and tradeoff are available elsewhere (see Barth et al. 2003; Xapsos et al. 1999, 2007; Cucinotta et al. 2010; Carnell et al. 2016), as is SEP forcasting (e.g. Kahler and Ling 2015; Laurenza et al. 2009). However, we do characterize the high-energy SEP spectra and their limits and spatial variations that affect radiation doses (e.g. Reames and Ng 1998; Reames 1999, 2013; Tylka and Dietrich 2009; Schrijver et al. 2012; Bruno et al. 2018). 


\subsection{High-Energy Spectra}

The single most important factor, in the dose of penetrating protons, may be the location of the high-energy spectral break or knee. A comparison of spectra in two events is shown in Fig. 6.1 where the contributions of "hard" and "soft" radiation boundaries are shown. The spectra in the two events are similar in the $10-100 \mathrm{MeV}$ region, partly controlled by the streaming limit (see Sects. 5.1.5 and 6.2). The spectrum of the April 1998 event (green) contributes mostly soft radiation in the region shaded yellow. The additional dose from the September 1989 SEP event is shaded red. Even behind $10 \mathrm{~g} \mathrm{~cm}^{-2}$ of material, astronauts would receive a dose of $40 \mathrm{mSv} \mathrm{h}^{-1}\left(\sim 4 \mathrm{rem} \mathrm{h}^{-1}\right.$, rem $=$ Roentgen equivalent for man $)$ at the intensities in the September 1989 event. The annual dose limit for a radiation worker in the United States is $50 \mathrm{mSv}$ (see review Cucinotta et al. 2010).

In Fig. 6.1, the proton spectral break or knee for the April 1998 event is about $40 \mathrm{MeV}$ while that for the September 1989 event is nearer $400 \mathrm{MeV}$. This spectral shape makes an important difference (see Sect. 3.4 and Fig. 3.7).

An extensive study of high-energy spectra in ground-level events (GLEs) has been conducted by Tylka and Dietrich (2009) who merged neutron-monitor data with satellite-based data. Two of these spectra are shown in Fig. 6.2. The authors

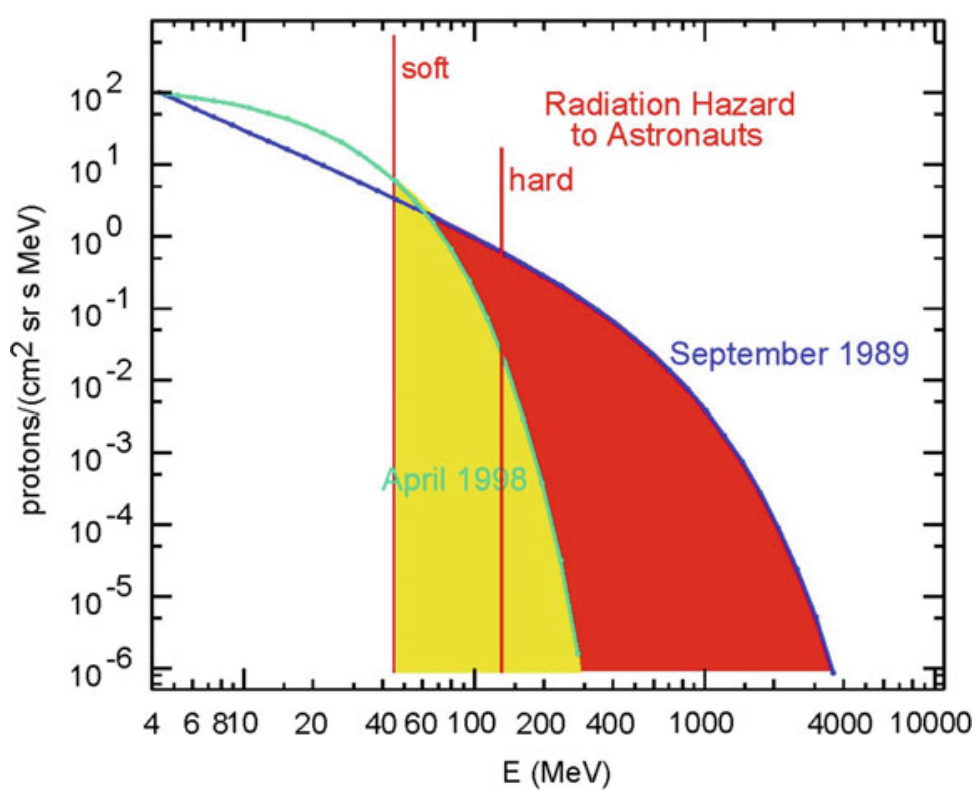

Fig. 6.1 Proton spectra in the SEP events of 20 April 1998 (green; based on Tylka et al. 2000) and 29 September 1989 (blue; based on Lovell et al. 1998) are compared. Typical energies of "soft" and "hard" radiation are shown. The hazardous portion of the spectrum of the April event is shaded yellow and the additional hazardous radiation from the September event is shaded red. (Reames 2013 (C) Springer) 

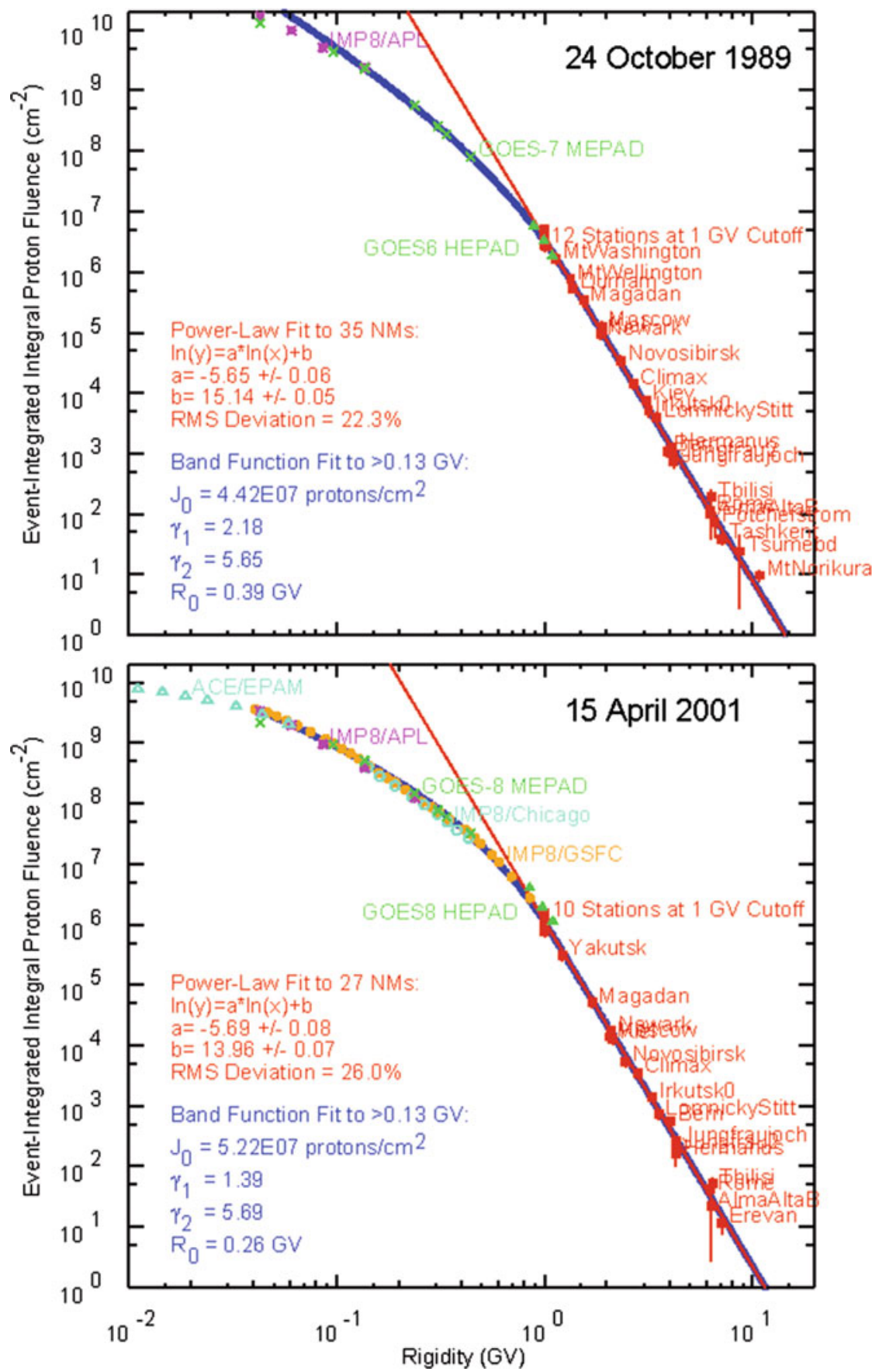

Fig. 6.2 Integral rigidity spectra are shown for two large GLEs. Cutoff rigidities for individual neutron-monitor stations (listed) are used; the spectra are corrected for neutron production vs. proton energy, and compared with the named satellite measurements. Fits to double power-law (Band) spectra are shown (Tylka and Dietrich 2009) 
construct integral rigidity spectra using the magnetic cutoff rigidity of the stations. They then correct for the fact that higher-energy protons produce increasingly more secondary neutrons, and they compare with satellite measurements. The spectra are then fit to the empirical double power-law (Band et al. 1993) spectra above 0.137 $\mathrm{GV}(10 \mathrm{MeV})$, for which the parameters are stated, and to a single power law in the neutron-monitor region. Note that the Cherenkov-radiation-based GOES/HEPAD instrument and the IMP/GSFC instrument overlap the neutron-monitor measurements extremely well up to rigidities above $1 \mathrm{GV}$.

Much of the neutron-monitor data have lain idle for 50 years. Tylka and Dietrich have performed a great service to finally find a way to analyze the data, compute spectra, and organize all of these data in a form that is useful for comparing and studying high-energy SEP events. Those responsible for determining the risk of radiation hazards to astronauts should certainly take advantage of this thorough study (see also Raukunen et al. 2018).

The fluence and the power-law fit above $1 \mathrm{GV}(430 \mathrm{MeV})$ for the GLEs from Tylka and Dietrich (2009) are shown in Fig. 6.3. The largest fluence is $4 \times 10^{6}$ protons $\mathrm{cm}^{-2} \mathrm{sr}^{-1}$ for the 23 February 1956 event, but the flattest spectra in the highenergy region are for the events of 7 May 1978 and 29 September 1979 (seen also in Figs. 6.1 and 6.5) events with rigidity spectral indices near 4.0. Most of the GLEs have rigidity spectral indices between 5 and 7 .

Parameters of these double power-law fits to GLE rigidity spectra have been tabulated by Raukunen et al. (2018) who also discuss interdependence of the fit parameters and fluence models based upon these results.

Recently, measurements of SEP proton spectra between $\sim 80 \mathrm{MeV}$ and a few GeV by the Payload for Antimatter Matter Exploration and Light-nuclei Astrophysics (PAMELA) have been reported for events from December 2006 and September 2014 by Bruno et al. (2018). These authors successfully fit proton fluence spectra to the Ellison and Ramaty (1985) form $E^{-\gamma} \exp \left(-E / E_{0}\right)$ for 26 events. Bruno et al. (2018) find no qualitative difference between GLEs, sub-GLEs, and non-GLEs, which form a continuous distribution.

Afanasiev et al. (2018) have applied their shock acceleration model to the GLE of 17 May 2012. They find increased acceleration of GV protons in regions of the shock with high Mach numbers and stress the importance of velocity differences between upstream and downstream scattering centers. Quasi-perpendicular regions of the shock may be involved to produce the observed timing.

\subsection{The Streaming Limit}

Protons streaming out early in a SEP event generate resonant waves that throttle the flow of subsequent particles, trapping them near the source. The streaming limit (see Sect. 5.1.5) is a transport phenomenon placing an upper bound on equilibrium intensities early in events once the waves become established (Reames and $\mathrm{Ng}$ 1998, 2010; $\mathrm{Ng}$ et al. 2003, 2012). If we plot the probability of attaining a given intensity, i.e. the number of hours a given intensity is observed in $\sim 11$ years, as in 


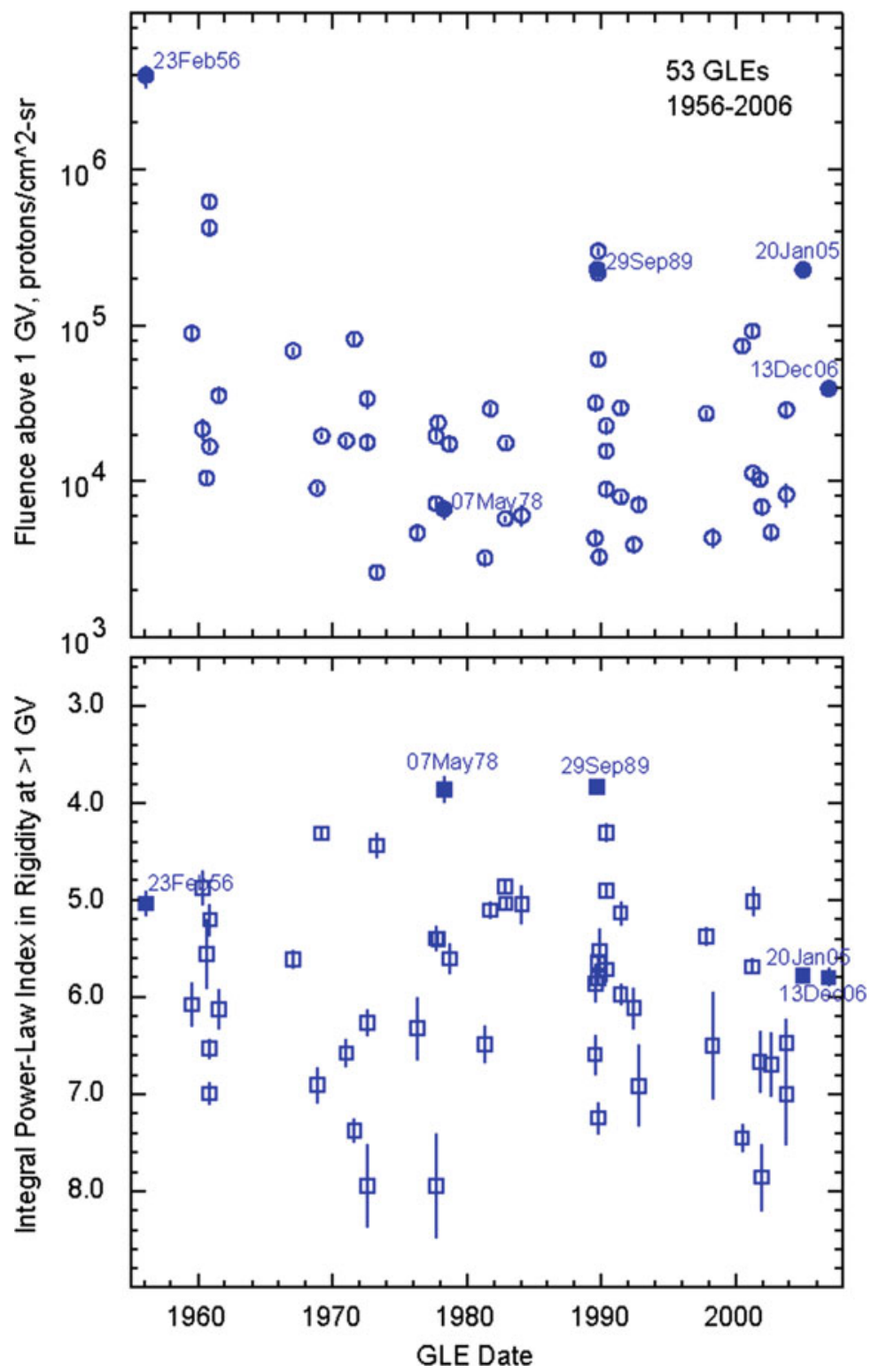

Fig. 6.3 The upper panel shows the proton fluence above $1 \mathrm{GV}(430 \mathrm{MeV})$ vs. time for each GLE. The lower panel shows the integral rigidity power-law spectral index also above $1 \mathrm{GV}$ (Tylka and Dietrich 2009; see also Raukunen et al. 2018) 


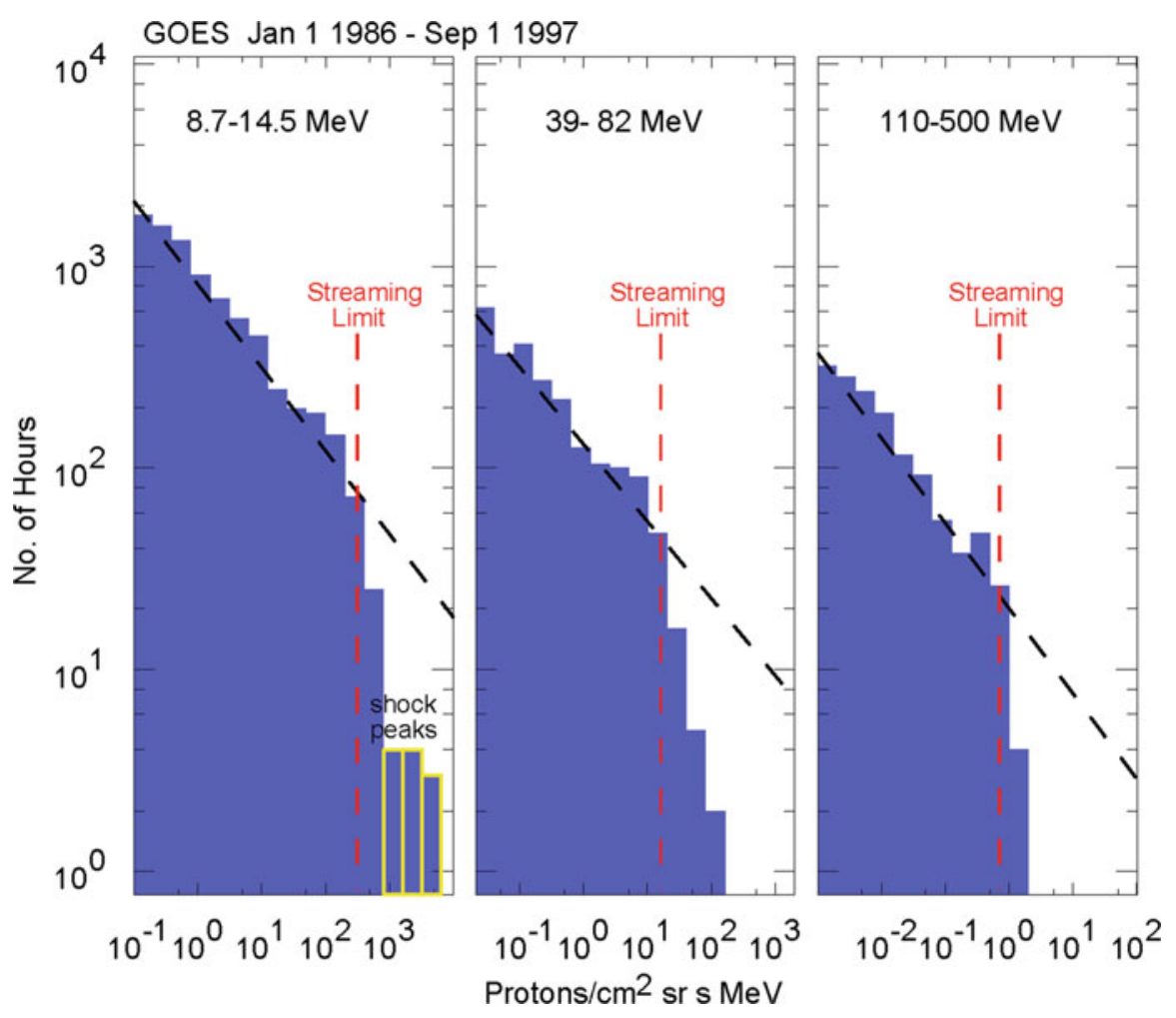

Fig. 6.4 The number of hours a given intensity is observed in $\sim 11$ years is shown for three different proton energy bins. Only intensities near the rarer shock peaks are seen above the streaming limit (Reames 2013 (C) Springer)

Fig. 6.4, we see a sudden drop above the streaming limit. Intensities near shock peaks are not limited by this mechanism since no net streaming is involved there. However, shock peaks occur late, when shocks have weakened and particles have spread spatially.

The black dashed lines in Fig. 6.4 are power-law fits below the streaming limit that decrease as the $\sim 0.4$ power of the intensity. This is often expressed as the differential slope, i.e. the rate of change in the number for a given change in the intensity, which decreases as the 1.4 power of the intensity in this case. Cliver et al. (2012) have compared different size measures of SEP events and of hard and soft solar X-ray events.

The streaming limit is not conveniently low so as to prevent excessive radiation exposure to astronauts, but at least it does offer a limit which is in force for a day or so before intensities begin to ramp up as the shock approaches. This allows astronauts time to reenter their vehicles and seek shelter, for example. The intensity level applied as the limits in Fig. 6.4 are shown during several large GLEs in Fig. 6.5. At the lower energies, up to $\sim 80 \mathrm{MeV}$, the peak at the shock can exceed the streaming limit by an order of magnitude or more. 


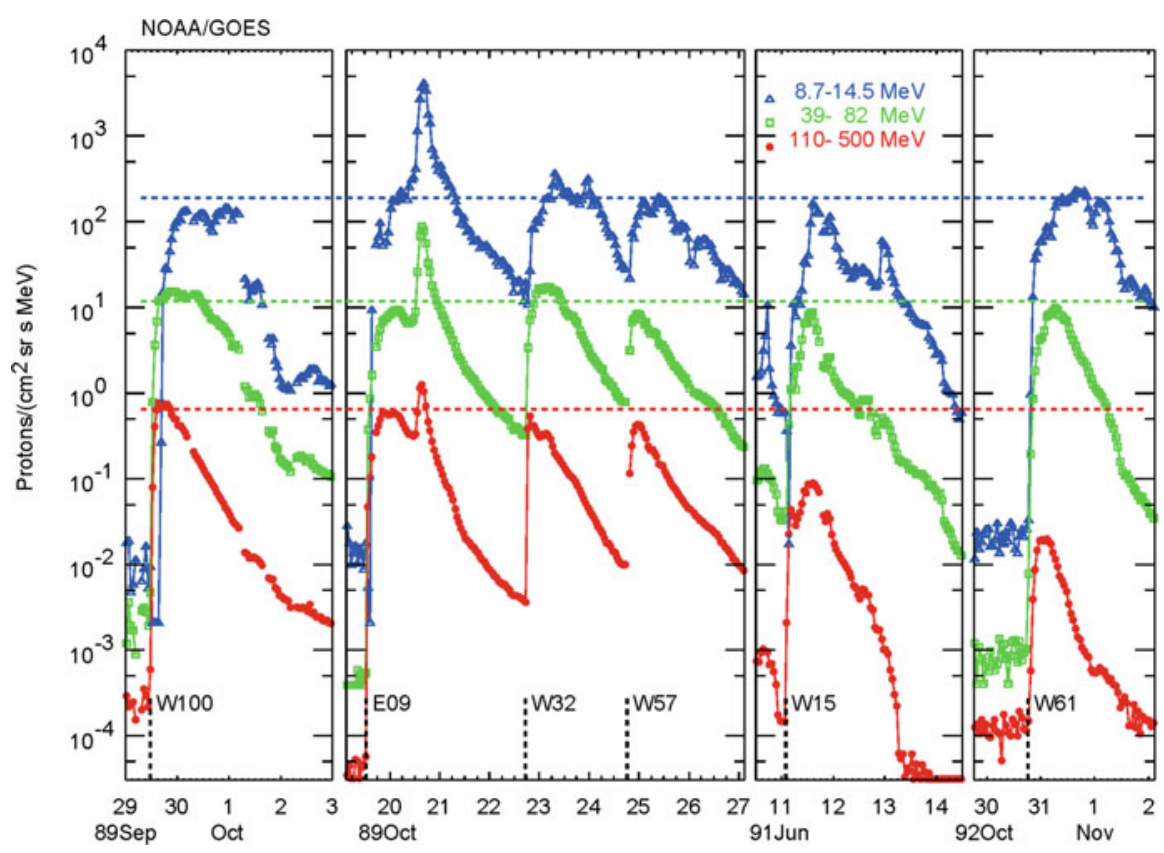

Fig. 6.5 Intensity levels are shown in six large SEP events with the corresponding streaming limits (Reames and Ng 1998 (C) AAS)

The rate of rise of the proton intensity can also be a factor in the establishment of equilibrium of the streaming limit as shown in Fig. 6.6. The fast rise of high-energy protons in the SEP event of 20 January 2005 allows the intensity to exceed the equilibrium limit until there has been sufficient wave growth to establish the equilibrium. Most events have slower evolution and do not overshoot the limit. Finally, Lario et al. (2009) have pointed out that trapping might also allow intensities to exceed the streaming limit.

It would be very useful to determine the rate of rare large SEP events and spikes of nitrates in ice cores were once suggested for this. However, these have been found to represent forest fires instead of SEP events (Schrijver et al. 2012).

\subsection{Radial Dependence}

Radial dependence of SEP intensities can be complex, but is often important for radiation assessment, especially on missions that approach the Sun. There is a wide variation in behavior. We might expect an impulsive injection to diverge like $r^{-3}$ while we have seen that reservoirs have no radial variation at all. Theoretically the dependence on space and time in a large gradual SEP event is shown in the illustrative example in Fig. 6.7. 

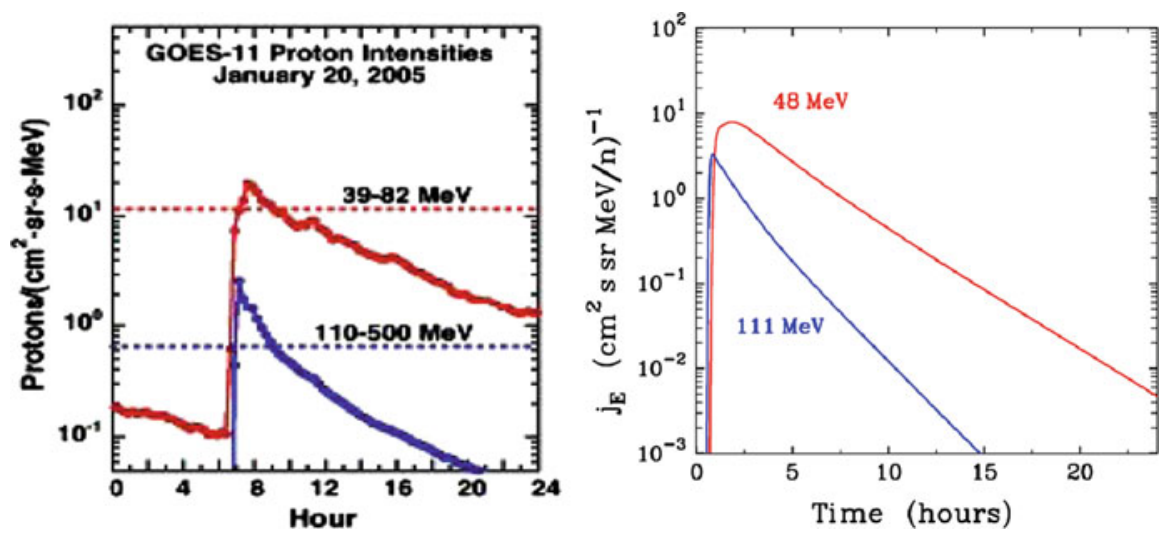

Fig. 6.6 The left panel shows that intensities in the event of January 20, 2005 briefly exceed the expected streaming limits from Fig. 6.5 (Mewaldt et al. 2007). The right panel shows that timedependent calculations described by $\mathrm{Ng}$ et al. (2012) also exceed these limits because there has not yet been enough proton flow to establish wave equilibrium at the highest energies. The fluence above $1 \mathrm{GV}$ for this event is compared with other events in Fig. 6.3

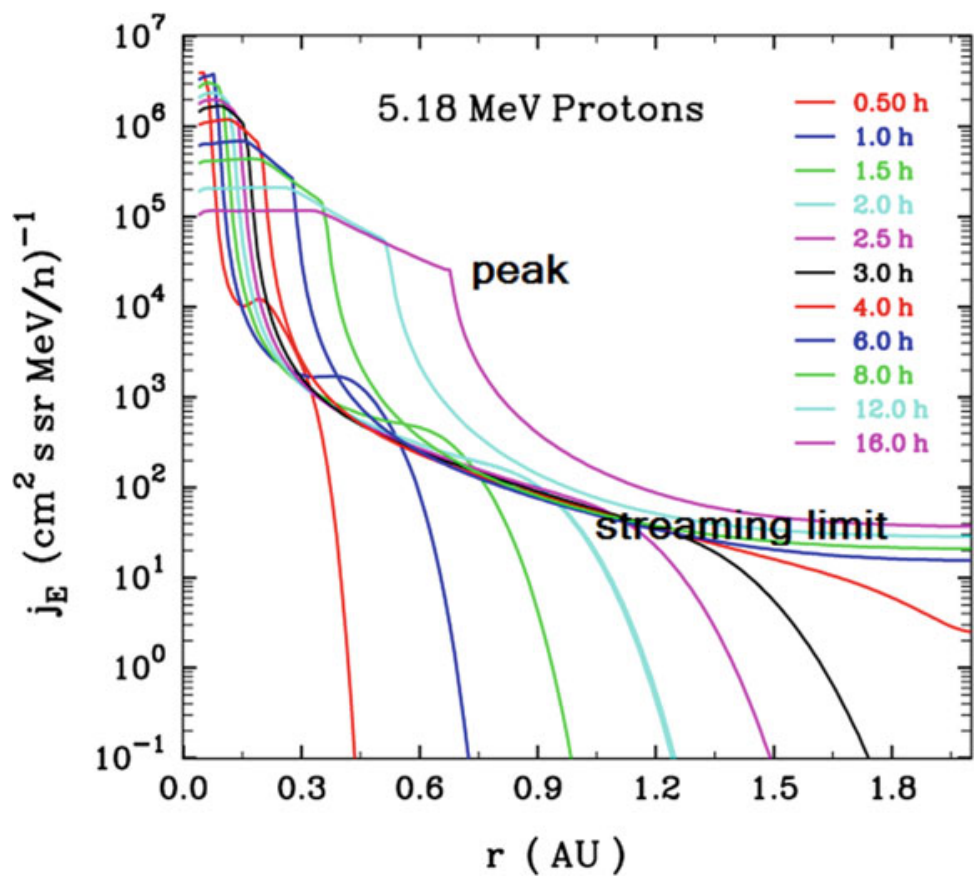

Fig. 6.7 Theoretical intensity of $5.18 \mathrm{MeV}$ protons vs. radius is shown as it varies with time during a large SEP event. Soon after arrival at a given radius, intensities rise to the streaming limit at that radius. At $1 \mathrm{AU}$, intensities are bounded near $\sim 100\left(\mathrm{~cm}^{2} \mathrm{~s} \mathrm{sr} \mathrm{MeV}\right)^{-1}$ until the shock reaches $\sim 0.7$ AU (see Ng et al. 2003, 2012). The tracks of the evolving "peak" and the lower "streaming limit" vs. $r$ are indicated 
Notice that the streaming limit is itself a strong function of radius and that the peak intensity at the shock follows a different radial track; both are the same at the shock when it is near the Sun. There can be a severe radiation hazard to equipment on a spacecraft that approaches the Sun. However, the probability may be small for occurrence of a large gradual SEP event during a brief passage of spacecraft perihelion. Early orbits of the Parker Solar Probe have reached inside 0.17 AU, but during the extreme quiet of solar minimum.

A model for calculating the radial dependences has been described by Verkhoglyadova et al. (2012).

\subsection{Radiation Hazards and an SEP Storm Shelter}

The NASA (2007; Carnell et al. 2016) permissible radiation exposure limits for humans in space are shown in Table 6.1. Entries in $\mathrm{mGy}-\mathrm{Eq}$ (milligray equivalent) have been multiplied by the relative biological effectiveness of the radiation ( 1.5 for protons, 2.5 for heavy ions) and are for short-term or career non-cancer effects $\left(1 \mathrm{~Gy}=1\right.$ joule $\mathrm{kg}^{-1}=100 \mathrm{rad}=6.24 \times 10^{12} \mathrm{MeV} \mathrm{kg}^{-1}$ deposited energy $)$. The NASA standard is an excess risk of exposure induced death from cancer of no more than $3 \%$ at the $95 \%$ confidence limit. Short term limits are used to prevent clinically significant non-cancer effects, such as acute radiation syndrome response, and degradations in crew performance (Townsend et al. 2018).

For human missions into deep space for extended periods, such as missions to the Moon or Mars, large SEP events become a significant radiation risk. Since it is impractical to shield an entire spacecraft from a SEP event, the idea of a temporary shelter that could be entered during a large SEP event has emerged as a possible alternative. It has even been suggested that such a shelter could be assembled from components that are already onboard (e.g. drinking water or even waste), but, of course, it must be possible to maintain and use this shelter for periods of several days. Water-filled vests have been proposed, but water-filled helmets may be as essential.

As a design standard, it has been proposed (Townsend et al. 2018) that the shelter should protect humans against a radiation dose based upon that encountered in the

Table 6.1 Permissible radiation exposure limits for humans

\begin{tabular}{l|l|l|l}
\hline Organ & $\begin{array}{l}30 \text { days } \\
(\mathrm{mGy}-\mathrm{Eq})\end{array}$ & $\begin{array}{l}1 \text { year } \\
(\mathrm{mGy}-\mathrm{Eq})\end{array}$ & $\begin{array}{l}\text { Career } \\
(\mathrm{mGy}-\mathrm{-Eq})\end{array}$ \\
\hline Lens & 1000 & 2000 & 4000 \\
\hline Skin & 1500 & 3000 & 6000 \\
\hline BFO & 250 & 500 & NA \\
\hline Heart & 250 & 500 & 1000 \\
\hline CNS & $500 \mathrm{mGy}$ & $1000 \mathrm{mGy}$ & $1500 \mathrm{mGy}$ \\
\hline CNS $(\mathrm{Z} \geq 10)$ & - & $100 \mathrm{mGy}$ & $250 \mathrm{mGy}$ \\
\hline
\end{tabular}

$B F O$ Blood-forming organs, CNS Central nervous system 


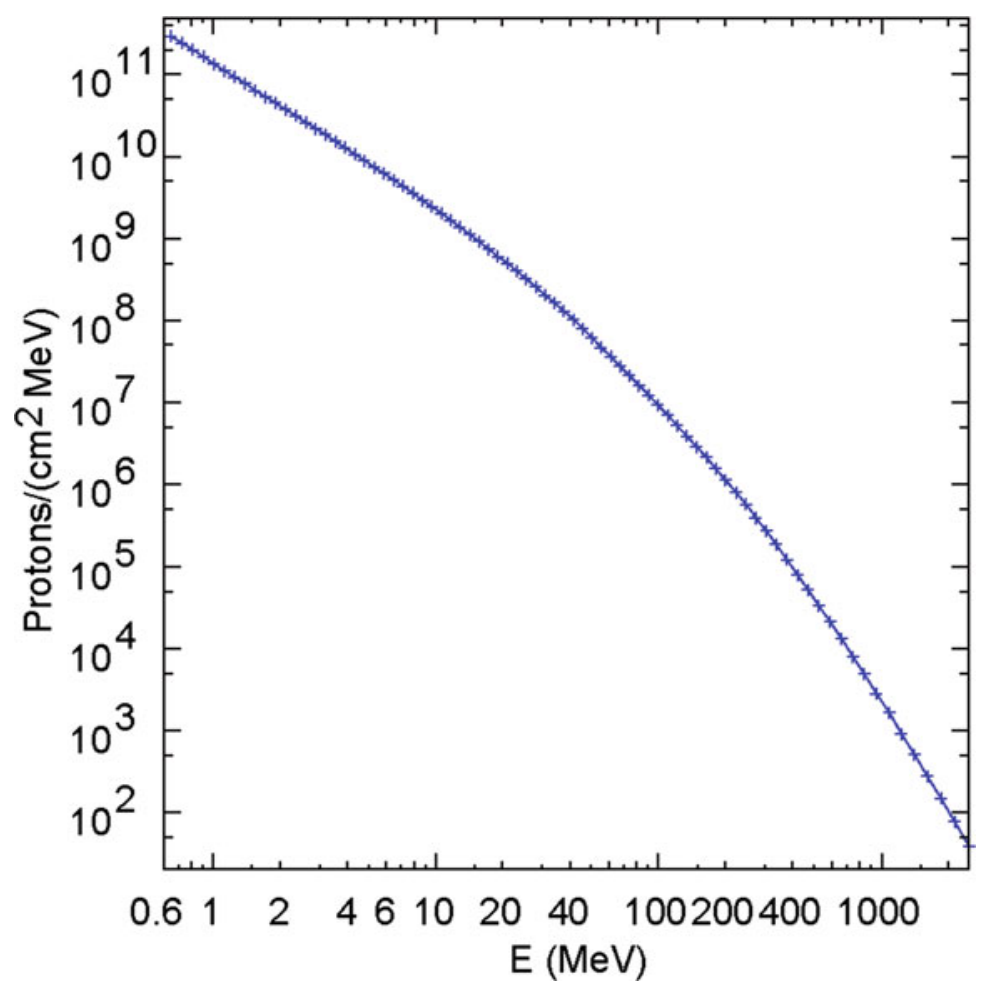

Fig. 6.8 The "Design Basis" SEP proton fluence spectrum proposed for shelter design (data from Townsend et al. 2018). This fluence may be distributed over several days (e.g. see Fig. 1.5b)

19 October 1989 SEP event series (see Fig. 6.5 or 1.5b) as is shown in Fig. 6.8 and tabulated in Townsend et al. (2018).

Typical BFO dose as a function of shielding thickness for $\mathrm{Al}$ and polyethylene are shown, for example, for female crew members in Fig. 6.9; similar curves for male crew members are also shown in Xapsos et al. (1999) and Townsend et al. (2018).

On planets or moons it may be possible to build efficient shelters out of local materials. In some cases, including the Earth's Moon, there may be caverns or caves, similar to lava tubes from volcanoes on Earth, which can provide a safe refuge.

\subsection{A Mission to Mars}

A mission to Mars beginning 26 November 2011 carried instruments that led to an estimate that the radiation dose during a $\sim 1$-year round-trip mission would be $660 \pm 12 \mathrm{mSv}$ (Zeitlin et al. 2013). Of course, this mission occurred during the notoriously weak Solar Cycle 24 that did not contain a SEP event like that of 23 February 1956 (see Fig. 6.3). Fortunately those events are very rare. 

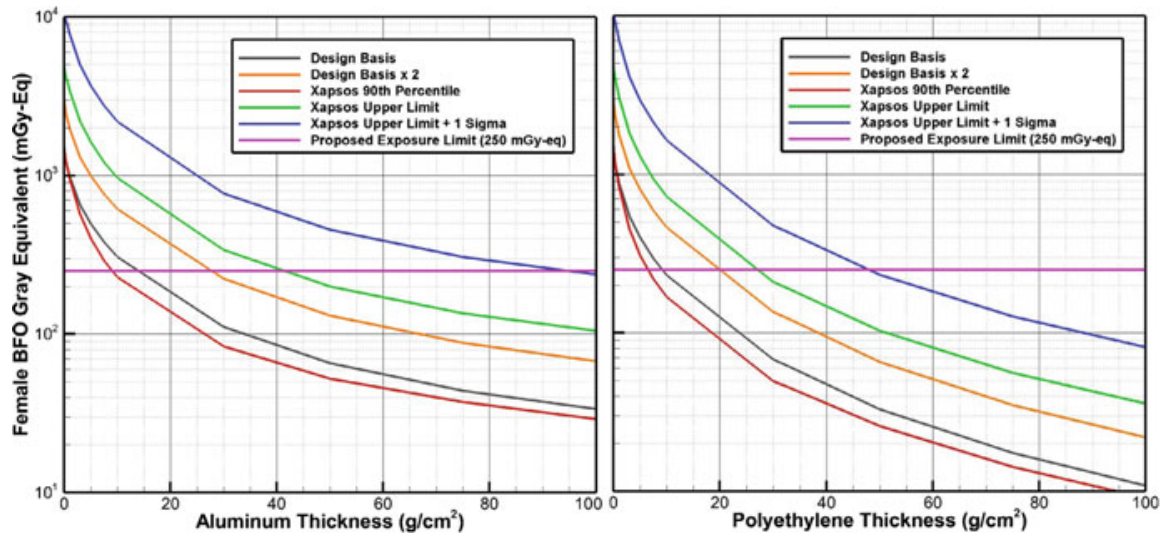

Fig. 6.9 The BFO radiation dose to female crew members is shown as a function of thickness of Al (left panel) and of polyethylene (right panel) for the energy spectra including the "Design Basis" spectrum in Fig. 6.8 (see Townsend et al. 2018; Xapsos et al. 1999). The figure shows that low-Z polyethylene is much more efficient than $\mathrm{Al}$

For the timing of a manned mission to Mars, one can go during solar maximum when SEP events are more probable but GCR intensities are reduced, or during solar minimum when SEPs are reduced but GCRs are at maximum (see Fig. 1.8). The continuous radiation of GCRs causes cancer risk in astronauts, while the SEPs pose a small risk of serious radiation sickness or even a fatal exposure. Most planning assumes a trip to Mars during solar maximum to reduce the cancer risk. It is assumed that SEP risk can be reduced somewhat by a safe-haven shelter with shielding of $20-40 \mathrm{~g} \mathrm{~cm}^{-2}$, combined with an adequate warning system. GCR radiation is not reduced by shielding; it is actually increased by production of secondary nuclearreaction products, including highly penetrating neutrals (Carnell et al. 2016).

Little effort is presently expended on studying the hazard from SEP events, i.e. assessing their risk of occurrence, and ensuring an appropriate structure is in place to provide adequate warning. In addition, there is little planning for contingencies in case of an extreme event. The probability of an extremely hazardous event occurring during a specific mission, even a 1-year mission, is relatively small, perhaps less than a few percent. The problem actually comes when there is a continuous human presence outside the Earth's magnetosphere; then it is not a question of if, but when.

\subsection{The Upper Atmosphere of Earth}

Ionization of the upper atmosphere during large SEP events can have significant long-term effects on the chemistry of the Earth's polar atmosphere. SEP ionization produces $\mathrm{HO}_{\mathrm{x}}$ and $\mathrm{NO}_{\mathrm{y}}$ in the mesosphere and stratosphere and the lifetime of the $\mathrm{NO}_{\mathrm{y}}$ allows it to affect ozone for months to years. Mesospheric ozone depletions of 
$50 \%$ can last for hours or days. Significant ozone depletions of $>10 \%$ can last a few months after SEP events. However, interference with the $\mathrm{Cl}$ - and $\mathrm{Br}$ - loss cycles actually caused an increase in total ozone, for example in 1992-1994, a few years after the October 1989 series of SEP events shown in Fig. 6.5 (Jackman et al. 2000, 2006). More recent events from January and March 2012 have also produced effects (von Clarmann et al. 2013) from this weaker solar cycle.

\subsection{SEPs and Exoplanets}

The possible effects of SEPs, stellar energetic particles, from other stars may also be an issue for the habitability and the development of life on exoplanets, or their moons (e.g. Lingam et al. 2018; Airapetian et al. 2019). SEPs could either constitute a radiation hazard in an otherwise habitable environment or they could induce reactions that produce the organic chemicals necessary for life. SEPs can have a significant effect on planetary atmospheres, depending upon planetary magnetic fields. In any case they are another factor that must be considered in the definition of a habitable zone.

The energy and intensity of energetic particles scales with the speed of CMEs and, while CMEs cannot be observed on other stars, their speed depends upon the intensity of the stellar magnetic field. That field is likely to be increased in young rapidly-rotating stars. Also, X-ray flares can be measured for stars and, while they are not directly related to SEPs, their number and intensity is related to the stellar magnetic field reconnection. Actually, the Sun is relatively inactive among stars of its class; it would be most interesting to see the range of SEP variation and its dependence upon stellar properties.

Fu et al. (2019) used the improved PATH model (Hu et al. 2017) to study the dependence of SEPs on the rotation rate of the star. They found that within $0.8 \mathrm{AU}$ for three times the solar rotation rate, the maximum particle energy at the shock front increased with the rotation rate of the star.

Detection of type II radio bursts could provide additional evidence of stellar SEPs. Unfortunately, radio emission below about $10 \mathrm{MHz}$ cannot be measured from the Earth's surface because of ionospheric interference.

\section{References}

Afanasiev, A., Vainio, R., Rouillard, A.P., Battarbee, M., Aran, A., Zucca, P.: Modelling of proton acceleration in application to a ground level enhancement. Astron.Astrophys. 614, A4 (2018). https://doi.org/10.1051/0004-6361/201731343

Airapetian, V.S., Barnes, R., Cohen, O., Collinson, G.A., Danchi, W.C., Dong, C.F., Del Genio, A. D., France, K., Garcia-Sage, K., Glocer, A et al.: Impact of space weather on climate and habitability of terrestrial type exoplanets (2019). arXiv: 1905.05093

Band, D., Matteson, J., Ford, L., Schaefer, B., Palmer, D., Teegarden, B., Cline, T., Briggs, M., Paciesas, W., Pendleton, G., et al.: BATSE observations of gamma-ray burst spectra. I - spectral diversity. Astrophys. J. 413, 281 (1993). https://doi.org/10.1086/172995 
Barth, J.L., Dyer, C.S., Stassinopoulos, E.G.: Space, atmospheric, and terrestrial radiation environments. IEEE Trans. Nucl. Sci. 50, 466 (2003). https://doi.org/10.1109/TNS.2003. 813131

Bruno, A., Bazilevskaya, G.A., Boezio, M., Christian, E.R., de Nolfo, G.A., Martucci, M., Merge', M., Mikhailov, V.V., et al.: Solar energetic particle events observed by the PAMELA mission. Astrophys. J. 862, 97 (2018). https://doi.org/10.3847/1538-4357/aacc26

Carnell, L., Blattnig, S, Hu, J. Huff, J., Kim, M.-H., Norman, R., Patel, Z., Simonsen, L., Wu, H.: NASA 1 Evidence Report: Risk of Acute Radiation Syndromes Due to Solar Particle Events. https://humanresearchroadmap.nasa.gov/evidence/reports/Acute.pdf?rnd=0. 543557888150009 (2016)

Cliver, E.W., Ling, A.G., Belov, A., Yashiro, S.: Size distributions of solar flares and solar energetic particle events. Astrophys. J. Lett. 756, L29 (2012). https://doi.org/10.1088/2041-8205/756/2/ L29

Cucinotta, F.A., Hu, S., Schwadron, N.A., Kozarev, K., Townsend, L.W., Kim, M.-H.Y.: Space radiation risk limits and Earth-Moon-Mars environmental models. Space Weather. 8, S00E09 (2010). https://doi.org/10.1029/2010SW000572

Ellison, D., Ramaty, R.: Shock acceleration of electrons and ions in solar flares. Astrophys. J. 298, 400 (1985). https://doi.org/10.1086/163623

Fu, S., Jiang, Y., Airapetian, V., Hu, J., Li, G., Zank, G.: Effect of star rotation rate on the characteristics of energetic particle events. Astrophys. J. 878(36) (2019). https://doi.org/10. 3847/2041-8213/ab271d

Hu, J., Li, G., Ao, X., Verkhoglyadova, O., Zank, G.: Modeling particle acceleration and transport at a 2D CME-driven shock. J. Geophys. Res. 122, 10,938 (2017). https://doi.org/10.1002/ 2017JA024077

Jackman, C.H., Fleming, E.L., Vitt, F.M.: Influence of extremely large solar proton events in a changing stratosphere. J. Geophys. Res. 105, 11659 (2000). https://doi.org/10.1029/ 2000JD900010

Jackman, C.H., Deland, M.T., Labow, G.J., Fleming, E.L., López-Puertas, M.: Satellite measurements of middle atmospheric impacts by solar proton events in solar cycle 23. Space Sci. Rev. 125, 381-391 (2006). https://doi.org/10.1007/s11214-006-9071-4

Kahler, S.W., Ling, A.: Dynamic SEP event probability forecasts. Space Weather. 13, 665 (2015). https://doi.org/10.1002/2015SW001222

Lario, D., Aran, A., Decker, R.B.: Major solar energetic particle events of solar cycles 22 and 23: intensities close to the streaming limit. Sol. Phys. 260, 407 (2009). https://doi.org/10.1007/ s11207-009-9463-1

Laurenza, M., Cliver, E.W., Hewitt, J., Storini, M., Ling, A.G., Balch, C.C., Kaiser, M.L.: A technique for short-term warning of solar energetic particle events based on flare location, flare size, and evidence of particle escape. Space Weather. 7, S04008 (2009). https://doi.org/10.1029/ 2007SW000379

Lingam, M., Dong, C., Fang, X., Jakosky, B.M., Loeb, A.: The propitious role of solar energetic particles in the origin of life. Astrophys. J. 853, 10 (2018). https://doi.org/10.3847/1538-4357/ aa9fef

Lovell, J.L., Duldig, M.L., Humble, J.E.: An extended analysis of the September 1989 cosmic ray ground-level enhancement. J. Geophys. Res. 103, 23,733 (1998). https://doi.org/10.1029/ 98JA02100

Mewaldt, R.A., Cohen, C.M.S., Haggerty, D.K., Mason, G.M., Looper, M.L., von Rosenvinge, T. T., Wiedenbeck, M.E.: Radiation risks from large solar energetic particle events. AIP Conf. Proc. 932, 277 (2007). https://doi.org/10.1063/1.2778975

NASA. NASA Space Flight Human System Standard. Volume 1: Crew Health, NASA-STD-3001. National Aeronautics and Space Administration, Washington. https://standards.nasa.gov/ standard/nasa/nasa-std-3001-vol-1 (2007) 
Ng, C.K., Reames, D.V., Tylka, A.J.: Modeling shock-accelerated solar energetic particles coupled to interplanetary Alfvén waves. Astrophys. J. 591, 461-485 (2003). https://doi.org/10.1086/ 375293

Ng, C.K., Reames, D.V., Tylka, A.J.: Solar energetic particles: shock acceleration and transport through self-amplified waves. AIP Conf. Proc. 1436, 212 (2012). https://doi.org/10.1063/1. 4723610

Raukunen, O., Vainio, R., Tylka, A.J., Dietrich, W.F., Jiggens, P., Heynderickx, D., Dierckxsens, M., Crosby, N., Ganse, U., Siipola, R.: Two solar proton fluence models based on ground level enhancement observations. J. Spa. Wea. Spa. Clim. 8, A04 (2018). https://doi.org/10.1051/ swsc/2017031

Reames, D.V.: Solar energetic particles: is there time to hide? Radiat. Meas. 30(3), 297 (1999). https://doi.org/10.1016/S1350-4487(99)00066-9

Reames, D.V.: The two sources of solar energetic particles. Space Sci. Rev. 175, 53 (2013). https:// doi.org/10.1007/s11214-013-9958-9

Reames, D.V., Ng, C.K.: Streaming-limited intensities of solar energetic particles. Astrophys. J. 504, 1002 (1998). https://doi.org/10.1086/306124

Reames, D.V., Ng, C.K.: Streaming-limited intensities of solar energetic particles on the intensity plateau. Astrophys. J. 722, 1286 (2010). https://doi.org/10.1088/0004-637X/723/2/1286

Schrijver, C.J., Beer, J., Baltensperger, U., Cliver, E.W., Güdel, M., Hudson, H.S., McCracken, K. G., Osten, R.A., Peter, T., Soderblom, D.R., Usoskin, I.G., Wolff, E.W.: Estimating the frequency of extremely energetic solar events, based on solar, stellar, lunar, and terrestrial records. J. Geophys. Res. 117, A08103 (2012). https://doi.org/10.1029/2012JA017706

Townsend, L.W., Adams, J.H., Blattnig, S.R., Clowdsley, M.S., Fry, D.J., Jun, I., McLeod, C.D., Minow, J.I., Moore, D.F., Norbury, J.W., Norman, R.B., Reames, D.V., Schwadron, N.A., Semones, E.J., Singleterry, R.C., Slaba, T.C., Werneth, C.M., Xapsos, M.A.: Solar particle event storm shelter requirements for missions beyond low earth orbit. Life Sci. in Space Res. 17, 32-39 (2018). https://doi.org/10.1016/j.lssr.2018.02.002

Tylka, A.J., Dietrich, W.F.: A new and comprehensive analysis of proton spectra in ground-level enhanced (GLE) solar particle events. In: Proceedings of 31st International Cosmic Ray Conference Lodz. http://icrc2009.uni.lodz.pl/proc/pdf/icrc0273.pdf (2009)

Tylka, A.J., Boberg, P.R., McGuire, R.E., Ng, C.K., Reames, D.V.: Temporal evolution in the spectra of gradual solar energetic particle events. In: Mewaldt, R.A., Jokipii, J.R., Lee, M.A., Möbius, E., Zurbuchen, T.H. (eds.) AIP Conference Proceedings 528, Acceleration and Transport of Energetic Particles Observed in the Heliosphere, p. 147. AIP, Melville (2000). https:// doi.org/10.1063/1.1324300

Verkhoglyadova, O.P., Li, G., Ao, X., Zank, G.P.: Radial dependence of peak proton and Iron ion fluxes in solar energetic particle events: application of the PATH code. Astrophys. J. 757, 75 (2012). https://doi.org/10.1016/j.physrep.2014.10.004

von Clarmann, T., Funke, B., López-Puertas, M., Kellmann, S., Linden, A., Stiller, G.P., Jackman, C.H., Harvey, V.L.: The solar proton events in 2012 as observed by MIPAS. Geophy. Res. Lett. 40, 2339 (2013). https://doi.org/10.1002/grl.50119

Xapsos, M.A., Summers, G.P., Barth, J.L., Stassinopoulos, E.G., Burke, E.A.: Probability model for worst case solar proton event fluences. IEEE Trans. Nucl. Sci. 46(6), 1481-1485 (1999). https://doi.org/10.1109/23.819111

Xapsos, M.A., Stauffer, C., Jordan, T., Barth, J.L., Mewaldt, R.A.: Model for cumulative solar heavy ion energy and linear energy transfer spectra. IEEE Trans. Nucl. Sci. 54, 1985 (2007). https://doi.org/10.1109/TNS.2007.910850

Zeitlin, C., Hassler, D.M., Cucinotta, F.A., Ehresmann, B., Wimmer-Schweingruber, R.F., Brinza, D.E., et al.: Measurements of energetic particle radiation in transit to Mars on the Mars Science Laboratory. Science. 340, 1080 (2013). https://doi.org/10.1126/science.1235989 
Open Access This chapter is licensed under the terms of the Creative Commons Attribution 4.0 International License (http://creativecommons.org/licenses/by/4.0/), which permits use, sharing, adaptation, distribution and reproduction in any medium or format, as long as you give appropriate credit to the original author(s) and the source, provide a link to the Creative Commons license and indicate if changes were made.

The images or other third party material in this chapter are included in the chapter's Creative Commons license, unless indicated otherwise in a credit line to the material. If material is not included in the chapter's Creative Commons license and your intended use is not permitted by statutory regulation or exceeds the permitted use, you will need to obtain permission directly from the copyright holder.

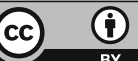

